\title{
Erhaltene sexuelle Funktion nach hochdosierter bildgestützter Protonentherapie des Prostatakarzinoms
}

\author{
Elisa Thomas ${ }^{1} \cdot$ Tobias Hölscher $^{1} \cdot$ Mechthild Krause $^{1}$ \\ Online publiziert: 20. Januar 2020 \\ (c) Der/die Autor(en) 2020
}

Hintergrund und Ziel der Arbeit Als Ziel setzten sich die Autoren die Identifikation prätherapeutischer Faktoren, um den Erhalt der Potenz nach einer hochdosierten Protonentherapie wegen Prostatakarzinom vorherzusagen.

Methode und Patienten In der prospektiven Kohortenstudie wurden die Potenzraten und die Auswirkung dieser auf die Lebensqualität mit Hilfe von etablierten Fragebögen (EPIC [Expanded Prostate Index Composite], IIEF [International Index of Erectile Function] und IPSS [International Prostate Symptome Score]) erfasst [1]. Von 1159 Patienten, die von August 2006 bis Mai 2010 eine hochdosierte Protonentherapie bei Prostatakarzinom erhalten hatten, wurden nach Ausschluss der Patienten mit Hormontherapie die Daten von 1005 Patienten ausgewertet. Appliziert wurde eine mediane Bestrahlungsdosis von $78 \mathrm{~Gy}\left(\mathrm{RBE}^{1}\right.$; 72-82 Gy [RBE]) in 36-39 Fraktionen. Außerdem wurden potenzbeeinflussende Komorbiditäten wie arterielle Hypertonie, Hyperlipidämie, Depression, Herzerkrankungen und eine entsprechende Medikation erfragt. Anschließend wurde anhand von Baseline-Faktoren ein Nomogramm zur Einschätzung der langfristigen Erhaltung der Potenz erstellt und anhand einer Patientenkohorte aus einer prospektiven Studie [2] validiert.

\footnotetext{
${ }^{1}$ Gy (RBE): Strahlendosis in Gy, korrigiert für die unterschiedliche relative biologische Wirksamkeit (RBE) der Partikeltherapie. Bei Protonen wird standardmäßig eine durchschnittliche RBE von 1,1 angenommen.
}

Originalpublikation Holtzman AL et al (2019) Patient-reported sexual survivorship following high-dose image-guided proton therapy for prostate cancer. Radiother Oncol 134:204-210

Prof. Dr. Mechthild Krause

mechthild.krause@uniklinikum-dresden.de

1 Klinik und Poliklinik für Strahlentherapie und Radioonkologie, Universitätsklinikum Carl Gustav Carus, Technische Universität Dresden, Fetscherstr. 74, 01307 Dresden, Deutschland
Ergebnisse Ausgewertet wurden 676 potente und 329 impotente Männer. Die Potenzraten der gesamten Kohorte betrugen $\mathrm{zu}$ Beginn der Behandlung 67\%, nach 6 Monaten $61 \%$, nach 2 Jahren $54 \%$ und nach 5 Jahren $46 \%$. Für die potenten Männer zeigten sich im Verlauf Potenzraten von $81 \%$ nach 6 Monaten, $68 \%$ nach 2 Jahren und $61 \%$ nach 5 Jahren. In einer univariaten Analyse mit Kovariatenstratifikation konnten folgende Faktoren, die die Potenz der 1005 Männer signifikant beeinflussen, identifiziert werden: der EPIC Sexual Summary Score, der IIEF sowie patientenbezogene Faktoren, wie Alter, eine vorliegende Herzerkrankung, Hypertonie, Hyperlipidämie und Diabetes mellitus. Der Body Mass Index (BMI), Beziehungsstatus, Ethnie, T-Stadium, Gleason-Score und PSA(Prostata-spezifisches Antigen)-Wert sowie die Bestrahlungsdosis beeinflussten hingegen die Potenz nicht statistisch signifikant. Durch rekursives Partitionieren wurde ein Nomogramm erstellt und die Patienten anhand von 2 Faktoren in 3 Patientenkategorien eingeteilt: eine Gruppe mit sehr guter Erektionsfähigkeit mit Potenzraten von $80 \%(n=137, p=0,83)$ nach 5 Jahren, eine intermediäre Gruppe mit Potenzraten um $62 \%$ ( $n=145, p=0,86)$ und eine Gruppe mit schlechten Potenzraten von $37 \%(n=117, p=0,19)$. Berücksichtigt wurde hierbei die Fähigkeit, eine Erektion auszubilden (mittels der einzelnen Frage EPIC Q57) sowie das Vorliegen einer (unspezifizierten) Herzerkrankung.

Schlussfolgerung der Autoren Die Erfassung der Potenz vor einer Strahlentherapie sowie der kardialen Begleiterkrankungen ermöglichen eine Vorhersage der erektilen Funktionsfähigkeit im Verlauf nach der Strahlentherapie. Das Miterfassen der Komorbiditäten im EPIC-Fragebogen könnte es dann ermöglichen, die Sexualität im Langzeitverlauf mittels Nomogrammen abzuschätzen und damit den Patienten bei der Wahl der Therapie zu helfen. 


\section{Kommentar}

Patienten, die eine Bestrahlung eines lokal begrenzten Prostatakarzinoms erhalten, weisen sehr gute Überlebenszeiten auf [3]. Aufgrund dessen ist das Auftreten von Spättoxizitäten, beispielweise Verlust der sexuellen Funktionsfähigkeit, für die Lebensqualität der Patienten äußerst relevant und damit ausschlaggebend für den Therapieentscheid. Um betroffenen Männern bei der Wahl der Therapie zu helfen, wurde im Rahmen der hier kommentierten Studie ein einfaches Nomogramm erstellt, um die 5-Jahres-Erektionsfähigkeit nach hochdosierter Strahlentherapie mit Protonen vorherzusagen. Männer mit antihormoneller Therapie wurden von der Studie ausgeschlossen. Validiert wurden die Daten anhand einer kleinen Patientenkohorte $(n=167)$, die eine hypofraktionierte Bestrahlung mit Protonen erhielten [2]. Dies lässt Vermutungen zu, dass das Nomogramm auch bei dieser letztgenannten Patientengruppe angewandt werden kann bzw. möglicherweise relativ robust ist gegenüber Änderungen im Fraktionierungsschema. Dennoch gilt zu bedenken, dass die Vergleichsgruppe klein ist. Eine zusätzlich externe Validierung wäre im Rahmen weiterer Studien wünschenswert.

Da nur Patienten mit einer Protonenbestrahlung untersucht wurden, wäre ein Vergleich mit Patienten nach Photonenbestrahlung sowie vor allem nach Operation oder kombinierter Operation und nachfolgender Bestrahlung sehr interessant. Ein Nomogramm, dass sowohl eine Abschätzung der erhaltenen Potenz nach Bestrahlung als auch nach Operation ermöglicht, wäre eine praktikable, einfache Methode, um die posttherapeutischen Nebenwirkungen vergleichbar abwägen zu können. Einen Unterschied in der Beeinflussung der Potenz würden wir zwischen einer Bestrahlung mit Photonen und mit Protonen nicht erwarten, da die Zielvolumina identisch sind.

In der PROSTQA-Studie wurden 2-Jahres-Potenzraten von $40 \%$ für operierte, $58 \%$ für bestrahlte und $63 \%$ für Patienten mit einer Brachytherapie festgestellt [4]. Im Vergleich dazu zeigte sich in der Gesamtkohorte der hier vorgestellten Studie eine Rate von $51 \%$ sowie bei den initial potenten Männern eine Rate von $68 \%$. Bei der Interpretation dieser guten Potenzraten gilt es zu beachten, dass es sich hierbei um ein gut selektiertes Patientenkollektiv handelt. Die potenten Männer waren im Mittel 64 Jahre und die impotenten 70 Jahre alt. Die univariate Analyse zeigte einen signifikanten Einfluss des Alters auf die Potenz der Männer. Dies konnte bereits in anderen Studien belegt werden [4]. Das junge Patientenalter sowie der Zugang zu einer Protonentherapie, welche zumindest im Herkunftsland der Studie in der Regel eher sozioökonomisch besser gestellten Patienten, denen meist eine gute medizinische Versorgung, z.B. auch mit Potenzmedikamenten, zur Verfügung steht, könnten mögliche Einflussfaktoren für die guten Potenzraten sein.

Die univariate Analyse zeigt außerdem, dass eine erhaltene Potenz vor allem von Komorbiditäten (Herzerkrankungen, Hypertonie, Hyperlipidämie, Diabetes mellitus) und einer prätherapeutisch erhaltenen Erektionsfähigkeit abhängt. Da mehrere Faktoren die Potenz signifikant beeinflussen, wäre an dieser Stelle eine multivariate Analyse aufschlussreich.

Interessanterweise konnte kein signifikanter Einfluss auf die Potenz durch tumorspezifische Faktoren (T-Stadium, Gleason-Score, PSA-Wert) sowie strahlentherapeutische Faktoren (Bestrahlungsdosis) festgestellt werden. Im Gegensatz dazu fand man in einer prospektiven, multizentrischen Studie [5, 6] einen Zusammenhang von Potenz und der eingestrahlten Dosis im Bulbus penis. Eine Dosis von über 52,5 Gy erhöhte die Impotenzraten signifikant. Erklärung für die differenten Ergebnisse könnte die applizierte Bestrahlungsdosis sein, da die mediane Dosis bei $14 \mathrm{~Gy}$ $(<1-73 \mathrm{~Gy}$ ) lag und weniger als $2 \%$ eine Dosis von 52,5 Gy am Bulbus penis erreichten.

\section{Fazit}

Die Studie umfasst die größte Kohorte zur sexuellen Funktion von Patienten, die eine Protonenbestrahlung der Prostata erhalten hatten. Das erstellte Nomogramm stellt eine einfache und schnelle Möglichkeit dar, die langfristig erhaltene Potenz nach Protonentherapie abzuschätzen. Um das Nomogramm in der Klinik anwenden zu können, wäre eine externe Validierung notwendig sowie die Berücksichtigung weiterer beeinflussender Faktoren.

Elisa Thomas, Tobias Hölscher, Mechthild Krause,

\section{Dresden}

Funding Open Access funding provided by Projekt DEAL.

Interessenkonflikt E. Thomas, T. Hölscher und M. Krause geben an, dass kein Interessenkonflikt besteht.

Open Access Dieser Artikel wird unter der Creative Commons Namensnennung 4.0 International Lizenz veröffentlicht, welche die Nutzung, Vervielfältigung, Bearbeitung, Verbreitung und Wiedergabe in jeglichem Medium und Format erlaubt, sofern Sie den/die ursprünglichen Autor(en) und die Quelle ordnungsgemäß nennen, einen Link zur Creative Commons Lizenz beifügen und angeben, ob Änderungen vorgenommen wurden.

Die in diesem Artikel enthaltenen Bilder und sonstiges Drittmaterial unterliegen ebenfalls der genannten Creative Commons Lizenz, sofern sich aus der Abbildungslegende nichts anderes ergibt. Sofern das betreffende Material nicht unter der genannten Creative Commons Lizenz steht und die betreffende Handlung nicht nach gesetzlichen Vorschriften erlaubt ist, ist für die oben aufgeführten Weiterverwendungen des Materials die Einwilligung des jeweiligen Rechteinhabers einzuholen.

Weitere Details zur Lizenz entnehmen Sie bitte der Lizenzinformation auf http://creativecommons.org/licenses/by/4.0/deed.de. 


\section{Literatur}

1. Holtzman AL et al (2019) Patient-reported sexual survivorship following high-dose image-guided proton therapy for prostate cancer. Radiother Oncol 134:204-210

2. Henderson RH et al (2017) Five-year outcomes from a prospective trial of image-guided accelerated hypofractionated proton therapy for prostate cancer. Acta Oncol 56:963-970

3. Hamdy FC et al (2016) 10-year outcomes after monitoring, surgery, or radiotherapy for localized prostate cancer. N Engl J Med 375(15):1415-1424
4. Alemozaffar M et al (2011) Prediction of erectile function following treatment for prostate cancer. JAMA 306:1205-1214

5. Roach M et al (2010) Radiation dose-volume effects and the penile bulb. Int J Radiat Oncol Biol Phys 76:S130-4

6. Roach M et al (2004) Penile bulb dose and impotence after threedimensional conformal radiotherapy for prostate cancer on RTOG 9406: findings from a prospective, multiinstitutional, phase I/II dose-escalation study. Int J Radiat Oncol Biol Phys 60:1351-1356 